\title{
Öffnungsklauseln - Instrument zur Krisenbewältigung oder Steigerung der Wettbewerbsfähigkeit?
}

Öffnungsklauseln gehören seit einiger Zeit zum Standardrepertoire tarifvertraglicher Regulierung. Entstanden vor dem Hintergrund eines vermeintlich zu starren und deshalb erodierenden Tarifsystems, dienen sie mittlerweile nicht nur zur Bewältigung betrieblicher Krisen, sondern auch zur generellen Stärkung der Wettbewerbsfähigkeit. Obwohl tarifliche Öffnungsklauseln auf ein breites (wissenschaftliches) Interesse stoßen, ist bislang noch nicht hinreichend geklärt, unter welchen betrieblichen Umständen von der mit ihnen verbundenen Option Gebrauch gemacht wird, kollektiv vereinbarte Standards zu unterschreiten. Dieser Frage wird mit den Daten des IAB-Betriebspanels 2007-2011 nachgegangen.

PETER ELLGUTH, SUSANNE KOHAUT

\section{Einleitung: Kontrollierte Dezentrali- sierung durch Öffnungsklauseln}

Das deutsche System der Arbeitsbeziehungen wird durch die gesetzlichen Bestimmungen zur Tarifautonomie und die betriebliche Interessenvertretung durch Betriebsräte getragen. Überbetriebliche Branchen- oder Flächentarifverträge spielen bei der Regelung von Arbeitsbedingungen und bei der Lohnfindung eine wesentliche Rolle. Im Arbeitsrecht haben Tarifverträge Vorrang gegenüber Betriebsvereinbarungen und Einzelarbeitsverträgen und können deshalb auch als Mindestarbeitsbedingungen interpretiert werden.

Allerdings gelten Branchentarifverträge ihren Kritikern häufig als zu starr, da sie branchenweit zwischen Arbeitgeberverbänden und Gewerkschaften abgeschlossen werden. Die spezifische Situation jedes einzelnen Betriebs kann somit nicht berücksichtigt werden. Deshalb stehen die Branchentarifverträge schon seit Langem immer wieder in der Kritik. Um unterschiedlichen betrieblichen Gegebenheiten Rechnung zu tragen, wurde in den vergangenen 30 Jahren über sogenannte Öffnungsklauseln nach und nach eine Vielzahl von Flexibilisierungs- und Differenzierungsmöglichkeiten in den Tarifverträgen eingeführt - ohne die Vorteile einer Flächentarifbindung ganz aufzugeben. Anfangs wurde dieses Instrument heftig und kontrovers diskutiert, da es bei Öffnungsklauseln immer um Abweichungen von tariflichen Standards nach unten geht, also zulasten der be- troffenen Arbeitnehmer. Den Unternehmen wiederum soll die Möglichkeit eröffnet werden, auf besondere betriebliche Situationen reagieren zu können. Inzwischen ist die Debatte abgeflaut und einer eher nüchternen Betrachtung gewichen. Öffnungsklauseln gehören mittlerweile zum Standard der tarifvertraglichen Regulierung.

Schon seit Mitte der 1980er Jahre spielen Öffnungsklauseln bei der Regelung der Arbeitszeit und ihrer Flexibilisierung eine wichtige Rolle. Sie wurden zunächst als Kompensation für die von den Gewerkschaften durchgesetzten tariflichen Arbeitszeitverkürzungen in der westdeutschen Metallindustrie eingeführt. Inzwischen sehen viele Flächentarifverträge flexible Regelungen zur Arbeitszeit vor. Sehr häufig wird die Möglichkeit eingeräumt, die Arbeitszeit ungleichmäßig auf Tage, Wochen oder Monate zu verteilen. Der Ausgleichszeitraum, in dem die durchschnittliche Arbeitszeit erreicht sein muss, beträgt meist zwölf Monate.

Die Anpassungskrise in den neuen Bundesländern und die Wirtschaftskrise in Westdeutschland Mitte der 1990er Jahre führten nach Bispinck (2004) zur zweiten Etappe der Flexibilisierung der Branchentarifverträge, in der zunehmend Öffnungsklauseln auch für Tarifentgelte vereinbart wurden, wobei es unterschiedliche Ausgestaltungen gibt. ${ }^{1}$ Trotz

1 Für ausführlichere Darstellungen von Öffnungsklauseln samt Typisierungen und Fallbeispielen sei auf Schnabel (2003, S. 82ff.) oder Bispinck/WSI-Tarifarchiv (2004) verwiesen. 
ihrer Vielfalt waren Entgeltöffnungsklauseln lange Zeit weniger verbreitet als Arbeitszeitöffnungsklauseln (Heinbach 2005). Sie sind ursprünglich in wirtschaftlich schwierigen Zeiten zur unmittelbaren Entlastung der Betriebe von Lohnkosten eingeführt worden. Für die Beschäftigten ist deren betriebliche Umsetzung i.d.R. mit größeren Zumutungen verbunden als die Nutzung von Arbeitszeitöffnungsklauseln. Damit dürften sie auch schwerer durchsetzbar sein. Hinzu kommt, dass ein Absenken von Löhnen oder Lohnbestandteilen immer mit einer negativen Signalwirkung für den Betrieb verbunden ist, die dazu führen kann, dass besonders qualifizierte Mitarbeiter das Unternehmen verlassen. Auch die Einstufung der Kreditwürdigkeit des Betriebs durch Banken kann dadurch negativ beeinflusst werden, was unter Umständen die Geldbeschaffung deutlich verteuert und so die wirtschaftliche Lage des Betriebs zusätzlich belastet (Franz/ Pfeiffer 2003, S. 34).

Es gibt mittlerweile eine Reihe wissenschaftlicher Arbeiten zum Thema tarifliche Öffnungsklauseln, die sich neben der Historie, den Regelungsbeständen und Einsatzbedingungen sowie der Ausgestaltung von Öffnungsklauseln vor allem mit deren Auswirkungen beschäftigen (vgl. Abschnitt 2). Eine Analyse über die Systematik der Nutzung von Öffnungsklauseln fehlt allerdings. Mit Daten des Betriebspanels des Instituts für Arbeitsmarkt- und Berufsforschung (IAB) soll deshalb hier der Frage nachgegangen werden, wodurch sich Betriebe auszeichnen, die auf tarifvertraglich vorhandene Öffnungsklauseln zurückgreifen und unter welchen Voraussetzungen sie diese nutzen. In diesem Zusammenhang interessiert insbesondere, ob es tatsächlich wirtschaftliche Krisensituationen sind, die zur betrieblichen Nutzung von Öffnungsklauseln führen, oder eher allgemeine Bestrebungen zur Verbesserung der Wettbewerbsfähigkeit.

\section{Stand der Forschung}

Bislang wurden Bedingungen für die betriebliche Nutzung von Öffnungsklauseln empirisch noch relativ selten untersucht. Kohaut/Schnabel (2007) analysieren, welche Faktoren Betriebe veranlassen, tarifliche Öffnungsklauseln in Anspruch zu nehmen. In dieser Studie werden Entgelt- und Arbeitszeitöffnungsklauseln unterschieden und jeweils getrennte ProbitModelle geschätzt. Die Ergebnisse zeigen, dass Betriebe mit sehr guter oder guter Ertragslage seltener von irgendeiner Art von Öffnungsklauseln Gebrauch machen. Hingegen werden Entgeltöffnungsklauseln von Betrieben mit schrumpfender Belegschaft häufiger genutzt. Die für die Analysen herangezogenen Daten des IAB-Betriebspanels 2005 enthalten zwar Informationen zum Vorhandensein und zur Nutzung von Öffnungsklauseln. Der Zeitpunkt des Einstiegs in die Nutzung von Öffnungsklauseln im Betrieb wird aber nicht erfasst. Für die Schätzung ergeben sich damit insofern Probleme, als die Öffnungsklauseln möglicherweise bereits über einen längeren
Zeitraum genutzt wurden und unklar bleibt, ob die betrachteten Determinanten nicht selbst durch die Nutzung der Öffnungsklauseln beeinflusst sind (Kohaut/Schnabel 2007, S. 37).

Auch Heinbach/Schröpfer (2008) untersuchen Bedingungen, die zu einer betrieblichen Nutzung von Öffnungsklauseln führen, wobei hier nicht zwischen Arbeitszeit- und Entgeltöffnungsklauseln unterschieden wird. Die Autoren interessieren sich vor allem für den Einfluss der Exporttätigkeit eines Betriebs (als Maß für dessen Produktivität) auf die Wahrscheinlichkeit, eine Öffnungsklausel zu nutzen. Die Schätzmodelle zeigen eine etwas niedrigere Wahrscheinlichkeit für die Anwendung von Öffnungsklauseln bei Betrieben, die in EU-Mitgliedstaaten exportieren. Betriebe, die nicht exportieren, und solche, die in Staaten außerhalb der EU exportieren, unterscheiden sich allerdings hinsichtlich der Nutzung von Öffnungsklauseln nicht. Die Analysen werden ebenfalls mit dem IAB-Betriebspanel 2005 durchgeführt, allerdings nur für das verarbeitende Gewerbe Westdeutschlands.

Spindler (2007) betrachtet ebenfalls Determinanten der Einführung von Öffnungsklauseln, allerdings nicht auf betrieblicher, sondern auf Branchenebene. Mit Hilfe einer Vielzahl einzelner Indikatoren aus unterschiedlichen Datenquellen zeigt die Studie, dass die Einführung von Öffnungsklauseln in Zusammenhang mit der wirtschaftlichen Situation in der jeweiligen Branche steht.

Andere empirische Analysen beschäftigen sich mit dem Einfluss von Öffnungsklauseln auf Lohnhöhe (Ellguth et al. 2012) und Lohnspreizung (Garloff/Gürtzgen 2012) oder das Beschäftigungswachstum in Betrieben (Brändle/Heinbach 2013). ${ }^{2}$

Ein weiterer relevanter Forschungsstrang besteht aus Arbeiten zu sogenannten betrieblichen Bündnissen für Arbeit (betriebliche Vereinbarungen zur Beschäftigungs- und Standortsicherung), die eine spezielle Form der betrieblichen Umsetzung von Öffnungsklauseln darstellen. Ursprünglich als Mittel zur Rettung existenzgefährdeter Betriebe gedacht, wandelten sich betriebliche Bündnisse von reinen Notfallmaßnahmen zu Instrumenten, die auch der generellen Verbesserung der Wettbewerbsfähigkeit dienen (Massa-Wirth 2007, S. 93).

In der Gesamtschau geht der Forschungsstand von zwei unterschiedlichen betrieblichen Motivlagen für die Nutzung betrieblicher Bündnisse aus. Berthold et al. (2003) fassen diese Unterscheidung begrifflich als Krisen- und Innovationsbündnisse. Sie beschreiben die jeweiligen ökonomischen Notwendigkeiten, die den beiden Bündnisarten zugrunde liegen, und die entsprechenden betrieblichen Ausgangslagen. In ihrer Anwendung implizieren Krisenbündnisse eine (unmittelbare) Entlastung bei den Lohnkosten, während es bei Innovationsbündnissen eher um eine „flexible und schnelle

2 Ein allgemeiner Überblick über empirische Arbeiten zu Öffnungsklauseln wird bei Brändle et al. (2011, S. 168ff.) gegeben. 
Anpassung an sich permanent verändernde Marktbedingungen“" (Berthold et al. 2003, S. 8) geht. Wie empirische Analysen zeigen, erweist sich eine solche Differenzierung der betrieblichen Motivlagen für Krisen- und Wettbewerbsbündnisse als durchaus sinnvoll. Multivariate Analysen, in denen den Einflussfaktoren für den Abschluss betrieblicher Bündnisse nachgegangen wird, bestätigen, dass sich die Betriebe je nach Bündnismotiv im Vorfeld in ganz unterschiedlichen Situationen befinden (Ellguth/Kohaut 2008). ${ }^{3}$ Diese für die Betrachtung betrieblicher Bündnisse fruchtbare Differenzierung erscheint uns auch für eine Analyse tariflicher Öffnungsklauseln sinnvoll und lässt sich durch die Unterscheidung von entgelt- und arbeitszeitrelevanten Regelungen nachvollziehen.

\section{Datengrundlage und Untersuchungsgegenstand}

Zur Verbreitung von Öffnungsklauseln gibt es nur wenige quantitative Datenquellen. Geht es um deren betriebliche Umsetzung, so reduziert sich die Datenbasis auf das IABBetriebspanel und die Betriebsrätebefragung des Wirtschafts- und Sozialwissenschaftlichen Instituts (WSI). Letztere hat sich zwar in verschiedenen Jahren diesem Thema gewidmet, die Erhebung ist aber auf die Betriebe mit Betriebsrat ab 20 Beschäftigten beschränkt. Zudem handelt es sich nicht um Längsschnittdaten.

Damit verbleibt das IAB-Betriebspanel als Datenquelle, eine jährliche Befragung von ca. 15.500 Betrieben aller Branchen und Größenklassen in Deutschland. ${ }^{4}$ Sie wird seit 1996 in West- und Ostdeutschland durchgeführt und ist repräsentativ für alle Betriebe mit mindestens einem sozialversicherungspflichtig Beschäftigten. Das IAB-Betriebspanel ist als Längsschnitterhebung angelegt, d.h. es werden nach Möglichkeit dieselben Betriebe wie im Vorjahr befragt. Damit können betriebsindividuelle Veränderungen verfolgt werden.

Informationen zur Existenz von Öffnungsklauseln in dem für den Betrieb geltenden Tarifvertrag und zur tatsächlichen betrieblichen Nutzung entsprechender Regelungen liefert das IAB-Betriebspanel 2005, 2007 und 2011. In der jüngsten Abfrage wird in fünf Einzelfragen der Existenz und Nutzung entsprechender Regelungen nachgegangen. Ein Item bezieht sich auf die „Verkürzung oder Verlängerung der tariflich vereinbarten Wochenarbeitszeiten (auch im Rahmen von Arbeitszeitkorridoren)“; die lohnrelevanten Öffnungsklauseln werden mit drei Einzelitems, die wir zusammenfassen, abgefragt. Mit dieser Dichotomisierung soll die auf Basis der unterschiedlichen betrieblichen Einsatzlogiken getroffene Unterteilung in Arbeitszeit- und Lohnöffnungsklauseln für die empirische Analyse umgesetzt werden.

In diesem Beitrag werden nur Betriebe der bundesdeutschen Privatwirtschaft betrachtet. Der öffentliche Dienst bleibt außen vor, da die Entscheidung der Betriebe für eine Nutzung von Öffnungsklauseln unter Marktbedingungen interessiert. Zudem werden die Analysen auf Betriebe mit mindestens fünf Beschäftigten begrenzt. Ohne diese Größenbegrenzung könnte es zu Verzerrungen kommen, wenn der Betriebsrat in die Schätzungen mit einbezogen wird.

Hinsichtlich der zur Verfügung stehenden Daten ist noch Folgendes anzumerken: Vieles deutet darauf hin, dass die Angaben zur Existenz von Öffnungsklauseln in dem für den Betrieb geltenden Tarifvertrag im IAB-Betriebspanel problematisch sind. Konkret ist davon auszugehen, dass der Anteil der Betriebe, deren Tarifvertrag entsprechende Möglichkeiten vorsieht, (deutlich) unterschätzt wird. Diese Einschätzung stützt sich zum einen auf Auswertungen aus dem WSI-Tarifarchiv, wonach in allen wichtigen Tarifverträgen Öffnungsklauseln verankert sind, die damit mittlerweile quasi zum normalen Bestandteil des deutschen Tarifsystems gehören würden (Bispinck/Schulten 2011, S. 3). Zum anderen bestätigt ein Blick in die Tarifdatenbank des Statistischen Bundesamtes ${ }^{5}$ den Eindruck, dass es im (weit) überwiegenden Teil der dort aufgelisteten Tarifverträge irgendeine Form von Öffnungsklausel gibt.

Des Weiteren kommt ein im Institut für angewandte Wirtschaftsforschung (IAW) erstellter Datensatz zur Existenz von Öffnungsklauseln in Tarifverträgen (IAW-Öffnungsklauseldatensatz) zu einer deutlich umfassenderen Verbreitung solcher Regelungen (Heinbach 2005; Heinbach/ Schröpfer 2008). Ebenfalls in diese Richtung weisen die Ergebnisse der WSI-Betriebsrätebefragungen zur Anwendung von Öffnungsklauseln (Bispinck/Schulten 2011).

Über die Ursachen der vermeintlichen Untererfassung von Öffnungsklauseln im IAB-Betriebspanel lässt sich nur spekulieren. Betriebe informieren sich vermutlich erst dann über die Möglichkeiten, die der geltende Tarifvertrag eventuell bietet, wenn die betriebliche Situation eine Nutzung solcher Elemente nahelegt. Das Wissen um die Existenz von Öffnungsklauseln wäre somit stark mit deren Nutzung verknüpft, zumindest mit einem konkreten Nutzungsinteresse. Diese Einschätzung wird gestützt durch den hohen Anteil an Betrieben im IAB-Betriebspanel, bei denen das Wissen über tarifvertragliche Öffnungsklauseln mit deren konkreter Nutzung korreliert. Anders gewendet: Wer nicht

3 Auch andere Arbeiten unterscheiden Vereinbarungen zur Krisenbewältigung und solche zur Stärkung der Wettbewerbsfähigkeit. Allerdings liegt bei diesen Studien der Fokus auf der Wirkung, die betriebliche Bündnisse entfalten, nicht auf der Charakterisierung der entsprechenden Betriebe (Bellmann et al. 2008; Bellmann/Gerner 2012a; Bellmann/Gerner 2012b).

4 Das IAB-Betriebspanel wird vonTNS Infratest Sozialforschung erhoben. Für weitere Informationen zum IAB-Betriebspanel siehe Fischer et al. (2008) und Ellguth et al. (2013).

5 Vgl. https://www.destatis.de/DE/ZahlenFakten/GesamtwirtschaftUmwelt/VerdiensteArbeits-kosten/Tarifverdienste/ TDB/TDB/TarifdatenbankIF.html (letzter Zugriff: 16.04.2013) 
mit den tarifvertraglichen Standards hadert, weiß vermutlich auch weniger über die entsprechenden Möglichkeiten, die das Regelwerk zu deren Unterschreitung bietet.

Darüber hinaus lässt sich argumentieren, dass eine tarifliche Öffnungsklausel generell einen gewissen substanziellen Gehalt aufweisen muss, um die Aufmerksamkeit der betrieblichen Praktiker zu wecken. So könnte z. B. die Option zur bloßen Verschiebung einer Jahressonderzahlung unterhalb dieser Schwelle liegen. Es mag allein schon deshalb eine ganze Reihe von Öffnungsklauseln (bzw. Betrieben mit entsprechenden Regelungen in ihrem Tarifvertrag) geben, die in einer Betriebsbefragung gar nicht erwähnt werden.

Nach dieser Argumentation könnte sich unter der großen Gruppe der tarifgebundenen Betriebe, die angeben, dass in den für sie einschlägigen Tarifverträgen keine Öffnungsklauseln existieren, durchaus eine beträchtliche Anzahl befinden, die eigentlich zur Gruppe der Betriebe mit nicht genutzten Öffnungsklauseln gehören. Die Informationen zur Existenz von Öffnungsklauseln wären somit wenig zuverlässig. Allerdings sollten dem gegenüber die betrieblichen Angaben zur Nutzung von (substanziellen) Öffnungsklauseln auch im Lichte dieser Argumente durchaus valide sein.

\section{Die betriebliche Nutzung von Öffnungsklauseln}

Für eine erste Orientierung sollen einige Zahlen zur Verbreitung und Nutzung tariflicher Öffnungsklauseln des IABBetriebspanels 2011 präsentiert werden. Bei $28 \%$ der betrachteten Betriebe ist nach eigenen Angaben irgendeine Öffnungsklausel im Tarifvertrag verankert (Tabelle 1). Wenn das der Fall ist, nutzen fast drei Viertel dieser Betriebe auch diese Option(en). Auf alle tarifgebundenen Betriebe bezogen, machen gut $20 \%$ von Öffnungsklauseln Gebrauch, um von den Tarifstandards abzuweichen. Während die (wahrgenommene) Existenz von Öffnungsklauseln stark größenabhängig ist (Kleinbetriebe $25 \%$, Großbetriebe $62 \%$ ), bewegt sich deren Nutzung (bezogen auf die dazu berechtigten Betriebe) über die Größenklassen hinweg auf vergleichbarem Niveau, was insgesamt zu einer Spannweite im Einsatz dieser Instrumente zwischen $19 \%$ in Kleinbetrieben und $45 \%$ in Großbetrieben (bezogen auf alle Betriebe) führt.

Die Verteilung der Existenz von Öffnungsklauseln entlang der Betriebsgröße ist vermutlich auch Ausdruck der angesprochenen selektiven Wahrnehmung entsprechender tarifvertraglicher Regelungen in den Betrieben. Es ist natürlich möglich, dass in eher kleinbetrieblich strukturierten Branchen seltener Öffnungsklauseln in den Tarifverträgen vereinbart werden. Plausibel ist aber auch, dass kleinere Betriebe vermutlich weniger gut über die Möglichkeiten informiert sind, die in den Regelwerken verankert wurden. Dafür spricht auch, dass in der 2005er und 2007er Welle des IAB-Betriebspanels (als noch eingangs mit einer Filterfrage nach der Existenz von Öffnungsklauseln im Tarifvertrag gefragt wurde), die kleineren Betriebe deutlich überdurchschnittlich die ,weiß nichtOption" wählten (siehe Kohaut/Schnabel 2007, S. 36). ${ }^{6}$

Entgeltrelevante Öffnungsklauseln sind (mittlerweile) ähnlich weit verbreitet wie solche, die Arbeitszeitregelungen betreffen. Letztere werden aber über alle Größenklassen hinweg stärker genutzt. Wie sich aus Tabelle 1 erschließen

6 Der Anteil der Betriebe, die angeben, nicht über dieses Thema Bescheid zu wissen, ist 2011 wohl durch die veränderte Fragestellung drastisch zurückgegangen.

TABELLE 1

Verbreitung und Nutzung von Öffnungsklauseln nach Betriebsgröße 2011*

Anteil der Betriebe in Prozent (hochgerechnete Ergebnisse)

\begin{tabular}{lcc|c}
\hline & \multicolumn{3}{c}{ Entgeltöffnungsklauseln } \\
\hline & $\begin{array}{c}\text { vor- } \\
\text { handen }^{1}\end{array}$ & $\begin{array}{c}\text { davon: } \\
\text { genutzt }\end{array}$ & $\begin{array}{c}\text { Nutzung bezogen } \\
\text { auf alle Betriebe }\end{array}$ \\
\hline 5 bis 49 Beschäftigte & 15 & 62 & 9 \\
\hline 50 bis 199 Beschäftigte & 24 & 60 & 14 \\
\hline 200 bis 499 Beschäftigte & 33 & 51 & 17 \\
\hline 500 und mehr Beschäftigte & 38 & 54 & 21 \\
\hline Gesamt ( $\mathrm{n}=4164)$ & $\mathbf{1 7}$ & $\mathbf{6 1}$ & 10 \\
\hline $\begin{array}{l}\text { nachrichtl.: Anteil Beschäftigte } \\
\text { in diesen Betrieben }\end{array}$ & 27 & 58 & 16 \\
\hline
\end{tabular}

\begin{tabular}{|c|c|c|}
\hline \multicolumn{3}{|c|}{ Arbeitszeitöffnungsklauseln } \\
\hline $\begin{array}{c}\text { vor- } \\
\text { handen }\end{array}$ & $\begin{array}{c}\text { davon: } \\
\text { genutzt }\end{array}$ & $\begin{array}{l}\text { Nutzung bezogen } \\
\text { auf alle Betriebe }\end{array}$ \\
\hline 16 & 74 & 11 \\
\hline 31 & 75 & 24 \\
\hline 42 & 76 & 32 \\
\hline 54 & 71 & 38 \\
\hline 18 & 74 & 13 \\
\hline 37 & 74 & 28 \\
\hline
\end{tabular}

\begin{tabular}{|c|c|c|}
\hline \multicolumn{3}{|c|}{ Irgendeine Öffnungsklausel } \\
\hline $\begin{array}{c}\text { vor- } \\
\text { handen }^{1}\end{array}$ & $\begin{array}{c}\text { davon: } \\
\text { genutzt }\end{array}$ & $\begin{array}{c}\text { Nutzung bezogen } \\
\text { auf alle Betriebe }\end{array}$ \\
\hline 25 & 73 & 19 \\
\hline 42 & 72 & 30 \\
\hline 54 & 76 & 41 \\
\hline 62 & 73 & 45 \\
\hline 28 & 73 & 20 \\
\hline 47 & 75 & 35 \\
\hline
\end{tabular}

*Basis: tarifgebundene Betriebe der Privatwirtschaft ab 5 Beschäftigte, ohne Landwirtschaft und Organisationen ohne Erwerbszweck.

${ }^{1}$ Bezogen auf alle tarifgebundenen Betriebe der Privatwirtschaft ab 5 Beschäftigte.

${ }^{2}$ Bezogen auf die Betriebe mit Öffnungsklauseln in den Tarifverträgen.

Quelle: IAB-Betriebspanel (2011). 
lässt, nutzt nur ein kleiner Teil der Betriebe beide Arten von Öffnungsklauseln, wenn es der geltende Tarifvertrag zulässt.

Die größenabhängige Verteilung führt dazu, dass knapp die Hälfte der Beschäftigten in Betrieben tätig ist, deren Tarifverträge Öffnungsklauseln enthalten. Die hohe Implementierungsquote führt in Beschäftigtenperspektive zu einem Nutzeranteil von $35 \%$, d. h. ein gutes Drittel aller Beschäftigten ist dort tätig, wo Öffnungsklauseln tatsächlich angewendet werden.

Der uns hier interessierende Einstieg in die Nutzung von Öffnungsklauseln, also die betriebliche Entscheidung, entsprechende tarifvertragliche Regelungen in Anspruch zu nehmen, um kollektiv vereinbarte Standards zu unterschreiten, wird im IAB-Betriebspanel nicht direkt abgefragt. Informationen hierüber können jedoch aus einem Abgleich der Angaben aus der 2007er und 2011er Welle gewonnen werden. Als Betriebe, die Öffnungsklauseln einführen, werden solche definiert, die 2007 angeben, keine derartigen Möglichkeiten in Anspruch zu nehmen, dies 2011 aber tun.

Damit nutzen wir den Längsschnittcharakter des Datensatzes und betrachten nur noch die Betriebe, die sowohl 2007 als auch 2011 existiert haben. Für den Zweck, solche Grundgesamtheiten deskriptiv darzustellen, werden im IAB-Betriebspanel spezielle Längsschnitthochrechnungsfaktoren zur Verfügung gestellt (Fischer et al. 2008, S. 25ff.).

Insgesamt zeigt sich, gemessen an der Nutzung beider Arten von Öffnungsklauseln, im Querschnitt ein hoher Anteil an Betrieben, die im Untersuchungszeitraum erstmals hiervon Gebrauch machen (10 bzw. 13 \%) und ein vergleichsweise geringer Anteil an „Dauernutzern“, also Betriebe, die in beiden Jahren Öffnungsklauseln anwenden (jeweils $3 \%$; Tabelle 2). Dies deutet auf einen relativ hohen „Umschlag“ bei der betrieblichen Umsetzung von Öffnungsklauseln hin.

Ein kurzer Blick auf die größenspezifische Verteilung des „Einstiegsgeschehens“ verdeutlicht zudem, dass es zwar bei der Implementierung entgeltrelevanter Öffnungsklauseln mit $10 \%$ der Klein- und $14 \%$ der Großbetriebe kaum entspre- chende Unterschiede gibt, es aber bei denjenigen Betrieben, die zu beiden Zeitpunkten von entsprechenden Regelungen Gebrauch machen, anders aussieht. Hier bleiben die Kleinbetriebe deutlich hinter den Großbetrieben zurück, woraus geschlossen werden kann, dass die Nutzung solcher Öffnungsklauseln in kleineren Betrieben noch wesentlich kurzlebiger ist als in den größeren. Diese Bild zeigt sich tendenziell auch bei arbeitszeitrelevanten Öffnungsklauseln. Allerdings geschieht hier auch der Einstieg in deren Nutzung in Großbetrieben mit $24 \%$ deutlich häufiger als in Kleinbetrieben (12\%).

\section{5. Ökonometrische Analyse der Imple- mentierung von Öffnungsklauseln}

\subsection{Theoretische Überlegungen}

Der Rekurs auf die Literatur zu betrieblichen Bündnissen (Abschnitt 2) hat verdeutlicht, dass für die Analyse der betrieblichen Hintergründe einer Implementierung von Öffnungsklauseln eine separate Betrachtung von entgelt- und arbeitszeitrelevanten Regelungen sinnvoll ist, da sich beide Regelungsarten grundsätzlich hinsichtlich ihrer Zielrichtung und Wirkung unterscheiden.

Entgeltöffnungsklauseln sollen angesichts einer ökonomischen Krise unmittelbar kostensenkend wirken (Berthold et al. 2003, S. 4). Betriebe, die dieses Instrument nutzen, sind allerdings nicht unbedingt nur Profiteure der von den Beschäftigten erzwungenen Zugeständnisse. Sie laufen vielmehr auch Gefahr, einen Image- oder Vertrauensverlust bei Beschäftigten, Kunden und Geschäftspartnern zu erleiden (vgl. Abschnitt 1). Deswegen kann angenommen werden, dass Entgeltöffnungsklauseln nur von Betrieben genutzt werden,

TABELLE 2

Anwendung von Öffnungsklauseln nach Betriebsgröße zwischen 2007 und 2011*

Anteil der Betriebe in Prozent (hochgerechnete Ergebnisse)

\begin{tabular}{l|c|c|c|c}
\hline & \multicolumn{5}{c}{ Entgeltöffnungsklauseln } \\
\hline & $\begin{array}{c}\text { Dauer- } \\
\text { nutzer }\end{array}$ & $\begin{array}{c}\text { Kein } \\
\text { Einsteiger }\end{array}$ & Einsteiger & $\begin{array}{c}\text { Alle tarifgebundenen } \\
\text { Betriebe }\end{array}$ \\
\hline 5 bis 49 Besch. & 3 & 87 & 10 & 100 \\
\hline 50 bis 199 Besch. & 7 & 81 & 12 & 100 \\
\hline 200 bis 499 Besch. & 12 & 76 & 12 & 100 \\
\hline 500 und mehr Besch. & 14 & 72 & 14 & 100 \\
\hline Gesamt $(n=2359)$ & $\mathbf{3}$ & $\mathbf{8 6}$ & $\mathbf{1 0}$ & $\mathbf{1 0 0}$ \\
\hline
\end{tabular}

\begin{tabular}{|c|c|c|c|}
\hline \multicolumn{4}{|c|}{ Arbeitszeitöffnungsklauseln } \\
\hline $\begin{array}{l}\text { Dauer- } \\
\text { nutzer }\end{array}$ & $\begin{array}{c}\text { Kein } \\
\text { Einsteiger }\end{array}$ & Einsteiger & $\begin{array}{c}\text { Alle tarifgebundenen } \\
\text { Betriebe }\end{array}$ \\
\hline 3 & 85 & 12 & 100 \\
\hline 7 & 73 & 20 & 100 \\
\hline 12 & 70 & 19 & 100 \\
\hline 14 & 62 & 24 & 100 \\
\hline 3 & 83 & 13 & 100 \\
\hline
\end{tabular}

* Basis: 2007 und 2011 existierende Betriebe der Privatwirtschaft ab 5 Beschäftigte mit Tarifbindung, ohne Landwirtschaft und Organisationen ohne Erwerbszweck. Rundungsfehler möglich.

Quelle: IAB-Betriebspanel $(2007 ; 2011)$ 
die sich tatsächlich in einer wirtschaftlichen Notlage befinden oder sich zumindest unter starkem Kostendruck sehen.

Demgegenüber zielen Arbeitszeitöffnungsklauseln darauf ab, „ohne Vorliegen einer betrieblichen Notlage die Wettbewerbsposition nachhaltig zu verbessern“ (ebd., S. 8). Sie sind daher als Mittel zur (weiteren) Steigerung der betrieblichen Anpassungsfähigkeit an sich permanent verändernde Märkte zu betrachten. Zu vermuten ist, dass die mit Arbeitszeit-Öffnungsklauseln verbundenen Zumutungen für die Beschäftigten geringer sind bzw. von ihnen eher akzeptiert werden als Einschnitte bei der Entlohnung.

Über solche allgemeinen ökonomischen Überlegungen hinaus gibt es unseres Wissens keine differenzierten theoretischen Annahmen, welche Betriebe in die Nutzung von Öffnungsklauseln einsteigen. Auch die wenigen vorliegenden empirischen Untersuchungen liefern keine eindeutigen Hinweise, welche Determinanten für den Einstieg entscheidend sind. Folglich kann im Weiteren nur teilweise auf theoretische Ansätze zur Auswahl einzelner Variablen zurückgegriffen werden. Wir orientieren uns im Folgenden z. T. an der Literatur zu den betrieblichen Bündnissen für Arbeit und unterscheiden verschiedene Variablengruppen, die die betriebliche Situation vor der Anwendung von Öffnungsklauseln charakterisieren.

\subsubsection{Wirtschaftliche Lage und Wettbewerbsposition}

Zur Erklärung, welche Betriebe Öffnungsklauseln nutzen, spielt - wie vorab dargelegt - die wirtschaftliche Situation des Betriebs eine zentrale Rolle. In die Analyse wird deshalb eine Reihe von Indikatoren aufgenommen, die den ökonomischen Hintergrund vor dem Einstieg in die Nutzung einer Öffnungsklausel beschreiben sollen. Zunächst werden die Einschätzungen der Geschäftsleitung zu der Ertragslage im letzten Geschäftsjahr herangezogen. Diese Variable ist Ausdruck der subjektiven Zufriedenheit bzw. Unzufriedenheit der Geschäftsleitung mit der wirtschaftlichen Situation des Betriebs. Auch die Einschätzungen zum Stand der Beschäftigten in fünf Jahren werden in die Schätzungen aufgenommen, um einen Eindruck der entsprechenden Erwartungen der Betriebe zu bekommen. Entgeltöffnungsklauseln sollten eher in Betrieben mit negativen Einschätzungen oder bei großer Unsicherheit hinsichtlich der Entwicklung von Ertragslage und Beschäftigung auftreten. Bei Arbeitszeitöffnungsklauseln gehen wir davon aus, dass sie als Instrument zur Erweiterung der betrieblichen Flexibilisierungsspielräume wohl eher unabhängig von den Geschäftserwartungen eingeführt werden.

Zur Charakterisierung der betrieblichen Wettbewerbsposition werden mehrere Variablen berücksichtigt. Dies ist zunächst der Exportanteil am Geschäftsvolumen. Theoretisch wird argumentiert, dass Firmen, die auf internationalen Märkten agieren, härterer Konkurrenz ausgesetzt sind und ihnen zusätzlich Kosten in Zusammenhang mit den Exportaktivitäten (z.B. Transportkosten) entstehen (Bernard/Wagner 1997). Um langfristig wirtschaftlich erfolgreich zu sein, müssen diese Betriebe eine höhere Produktivität aufweisen. Dieser Zu- sammenhang kann auch empirisch bestätigt werden (Wagner 2007). Somit stellen exportierende Betriebe eher eine Positivauswahl dar, die im Vergleich zu solchen, die nicht exportieren, besser aufgestellt sind. Falls diese Überlegungen zutreffen, dann wäre zu erwarten, dass die Arbeitszeitöffnungsklauseln mit einer höheren Wahrscheinlichkeit in exportierenden Betrieben und Entgeltöffnungsklauseln eher in nicht exportierenden Betrieben eingeführt werden. Die vorliegenden empirischen Studien liefern bislang keine klaren Hinweise zum Einfluss des Exports auf die Nutzung von Öffnungsklauseln.

Als weitere Variable wird die Selbsteinschätzung der Betriebe zur Beurteilung des Konkurrenz- und Wettbewerbsdrucks, dem sie sich ausgesetzt sehen, berücksichtigt. Aus den fünfstufigen Angaben wird eine Dummyvariable erzeugt, die angibt, ob der Betrieb einem hohen Wettbewerbsdruck ausgesetzt ist.

Der wirtschaftliche Erfolg und die Anpassungsfähigkeit eines Betriebs hängen entscheidend vom technischen Fortschritt und dessen Umsetzung im Betrieb ab. Betriebe, die investieren und Innovationen umsetzen, können sich im Vergleich zu ihren Wettbewerbern besser im Markt behaupten und sind auch für eventuelle Veränderungen auf den Märkten besser gerüstet. Um dies abzubilden, werden Informationen $\mathrm{zu}$ Produktinnovationen in den letzten beiden Jahren und zu Investitionen im Vorjahr jeweils als Dummyvariablen in die Schätzungen einbezogen. Es wäre zu erwarten, dass innovative Betriebe und solche die investieren eher keine Entgeltöffnungsklauseln implementieren, da sie auf den technischen Fortschritt setzen. Die Implementierung von Arbeitszeitöffnungsklauseln wiederum sollte für diese Betriebe eher Teil einer Gesamtstrategie zur „Erschließung von Effizienzsteigerungspotenzialen“ (Berthold et al. 2003, S. 8) sein.

\subsubsection{Flexibilisierungsinstrumente}

Die Bedeutung betrieblicher Flexibilität für Wirtschaftswachstum und Wettbewerbsfähigkeit wird immer wieder betont (z. B. Hohendanner/Bellmann 2006). Eine zunehmende Globalisierung der Wirtschaft mit dem einhergehenden starken internationalen Wettbewerb, aber auch nationale Veränderungen wie der demografische Wandel erfordern eine ständige Anpassung der Betriebe an sich verändernde Bedingungen. Wie für alle anderen Betriebe, so steht auch für die an Branchentarifverträge gebundenen eine Reihe von Instrumenten zur Verfügung, die ihnen Flexibilitätsspielräume eröffnen.

Zur Anpassung der Beschäftigung haben Betriebe die Möglichkeit, entweder interne oder externe Flexibilisierungsformen zu nutzen (Dütsch/Struck 2007). Im ersten Fall werden Anpassungsmaßnahmen innerhalb des Betriebs bei den bestehenden Beschäftigungsverhältnissen vorgenommen (z. B. durch Überstunden oder flexible Arbeitszeitmodelle). Die externe Flexibilisierung erfolgt über den Markt, indem beispielsweise Leiharbeitskräfte eingestellt werden (Hohendanner/Bellmann 2006). Empirische Ergebnisse zeigen, dass interne und externe Personalanpassungsmaßnahmen komplementär eingesetzt werden (Dütsch/Struck 2007, S. 40). 
In die Schätzmodelle werden als sogenannte externe Flexibilitätsinstrumente der Anteil der befristet Beschäftigten, die Quote der freien Mitarbeiter und die Quote der Leiharbeiter in die Analyse einbezogen. Als internes Flexibilitätsinstrument wird darüber hinaus berücksichtigt, ob ein Betrieb seine Belegschaft weiterbildet. Wir gehen davon aus, dass sich in Betrieben, die diese Flexibilitätsinstrumente bereits anwenden, die Wahrscheinlichkeit eines Einstiegs in die Nutzung von Arbeitszeitöffnungsklauseln erhöht, da dadurch weiteres internes Flexibilitätspotenzial erschlossen wird, das eher komplementär als substitutiv eingesetzt wird. Nach dieser Logik ist ein gegenteiliger (negativer) Zusammenhang für die Anwendung von Entgeltöffnungsklauseln zu vermuten. Betriebe, die diese externen Anpassungsmaßnahmen nutzen, dürften sich eher nicht gezwungen sehen, die Lohnkosten zu reduzieren. Oder umgekehrt formuliert: Werden entsprechende Flexibilitätspotenziale nicht ausgeschöpft, könnte sich die Wahrscheinlichkeit, dass auf entgeltrelevante Öffnungsklauseln zurückgegriffen werden muss, erhöhen.

\subsubsection{Lohnkosten}

Besonders Lohnkosten stellen für viele Betriebe einen wesentlichen Kostenanteil dar. Tarifgebundene Betriebe können diesen nicht ohne Weiteres beeinflussen, da sowohl die Lohnhöhe als auch die Arbeitszeiten meist in den Tarifverträgen festgelegt sind. Über tarifliche Öffnungsklauseln können je nach Ausgestaltung entweder die Arbeitszeiten oder sogar die Löhne direkt vom Betrieb verändert werden, um die Personalkosten zu senken und so die Wettbewerbsfähigkeit des Betriebs zu verbessern.

Um diesen Einfluss der Lohnstruktur auf den Einstieg in die Nutzung von Öffnungsklauseln zu erfassen, werden in die Schätzung die logarithmierten Lohnkosten pro Vollzeitäquivalent, der Anteil der Lohnkosten am Umsatz und eine Dummyvariable mit aufgenommen, die angibt, ob ein Betrieb eine hohe Belastung durch Lohnkosten in den nächsten Jahren erwartet. Hier ist der erwartete Zusammenhang auf die Nutzung einer Entgeltöffnungsklausel positiv. Das heißt, je höher die Löhne bzw. je stärker die Belastung durch die Lohnkosten, desto wahrscheinlicher die Implementierung einer Entgeltöffnungsklausel, wohingegen die Nutzung von Arbeitszeitöffnungsklauseln davon eher unabhängig sein sollte.

Zusätzlich wird in den Schätzungen berücksichtigt, ob ein Betrieb übertariflich entlohnt. Eine Entlohnung über das im Tarifvertrag festgelegte Niveau hinaus kann von einem Betrieb in einer schwierigen wirtschaftlichen Lage abgebaut werden, ohne dass eine Öffnungsklausel hierfür genutzt werden müsste. Folglich ist eher ein negativer Einfluss auf die Umsetzung von Entgeltöffnungsklauseln zu erwarten.

\subsubsection{Betriebsrat}

In vielen tariflichen Öffnungsklauseln wird explizit auf den Betriebsrat als Instanz verwiesen, dem gemeinsam mit der Geschäftsleitung die Umsetzung der entsprechenden Rege- lungen in Form einer Betriebsvereinbarung obliegt. Häufig ist die Existenz eines Betriebsrates eine zwingende Voraussetzung, um von Öffnungsklauseln Gebrauch machen zu können (vgl. Massa-Wirth 2007, S. 124). Nur in einem Teil der Tarifverträge ist für betriebsratslose Betriebe ein alternatives Prozedere vorgesehen. Allein aufgrund dieser (teilweise exklusiven) Aufgabenzuweisung sollte es in Betrieben mit Betriebsrat mit einer höheren Wahrscheinlichkeit zur Implementierung jeglicher Art von Öffnungsklauseln kommen.

Auf der anderen Seite ist anzunehmen, dass der Betriebsrat (in Wahrnehmung seiner Schutzfunktion) seine Aufgabe darin sieht, die Beschäftigten vor allzu großen Zumutungen zu bewahren. Da ihm ebenso das Betriebswohl, sprich das Überleben und die Wettbewerbsfähigkeit des Betriebs am Herzen liegen sollte, könnte das dazu führen, dass er zwar Maßnahmen, die den Betrieb besser für den Wettbewerb rüsten, unterstützt und somit die Nutzung entsprechender arbeitszeitrelevanter Öffnungsklauseln nicht behindert. Die Vereinbarung von Lohnabschlägen könnte aber die Grenze des Zumutbaren überschreiten und den Widerstand des Betriebsrats hervorrufen (Ellguth et al. 2012).

\subsubsection{Kontrollvariablen}

Zuletzt finden noch mehrere Kontrollvariablen Berücksichtigung. Zunächst könnte der Standort des Betriebes eine wichtige Einflussgröße sein. Da im Osten der Bundesrepublik weiterreichende Öffnungsklauseln in den Tarifverträgen existieren und die Beschäftigten in Ostdeutschland aufgrund der schlechteren Arbeitsmarktlage als konzessionsbereiter gelten, wird er als Variable in die Schätzung aufgenommen (Massa-Wirth 2007, S. 95).

Die Aushandlung von Partizipations- und Interessensregulierungsformen sind immer auch von den „Organisationsund Verhandlungskulturen“ (Bosch et al. 1999, S. 29) der Betriebe abhängig. Betriebskulturelle Unterschiede können vor allem über die Betriebsgröße und die Art der Betriebsführung erfasst werden. Es ist zu erwarten, dass eigentümergeführte Betriebe eher informelle Regelungen bevorzugen. In managergeführten Betrieben sind formelle Strukturen und Regelungen notwendig, da die Manager ihre Entscheidungen Dritten gegenüber rechtfertigen müssen. Außerdem sind formelle Regelungen und Abläufe auch auf anderen Gebieten (z.B. der Bilanzierung) notwendig und damit der Geschäftsführung vertraut. Die Nutzung von Öffnungsklauseln, sowohl für Arbeitszeitfragen als auch zum Entgelt, dürfte somit wahrscheinlicher sein.

Allgemein wird argumentiert, dass in Branchentarifverträgen die Interessen kleinerer Betriebe nicht hinreichend berücksichtigt werden, da vor allem große Unternehmen Einfluss in den Verbänden und damit auf die Tarifverhandlungen haben. Daraus kann abgeleitet werden, dass es eher die kleineren Betriebe sein dürften, die Öffnungsklauseln nutzen. Um diesen Einfluss zu modellieren, wird in die Schätzungen die logarithmierte Anzahl der Beschäftig- 
ten aufgenommen. Auf diese Weise können auch eventuelle nicht lineare Zusammenhänge abgebildet werden.

In den Schätzungen wird darüber hinaus das Betriebsalter berücksichtigt, da bestehenden betrieblichen Strukturen eine gewisse Beharrungskraft zugeschrieben wird, aktuelle Entscheidungen also auch von ,gewachsenen“ betrieblichen Gegebenheiten abhängen können. Das Betriebsalter wird hier operationalisiert durch einen Dummy, der angibt, ob ein Betrieb vor 1990 gegründet wurde. Ältere Betriebe werden vermutlich Öffnungsklauseln häufiger einführen, da sie als eher strukturkonservativ gelten und möglicherweise Entwicklungen am Markt „verschlafen“ haben. Jüngere Betriebe müssen größere Anstrengungen unternehmen, um sich zu etablieren, und dürften deshalb auch näher an den aktuellen Entwicklungen des Marktes sein. Des Weiteren haben sie weniger rechtliche Restriktionen zu beachten (z. B. Sonderregelungen beim Kündigungsschutz) und können so flexibel reagieren, ohne dass sie Öffnungsklauseln nutzen müssen.

Zuletzt wird die Branchenzugehörigkeit der Betriebe als Dummyvariable in das Modell aufgenommen. So können branchenspezifische Besonderheiten berücksichtigt werden.

\subsection{Schätzstrategie}

Der Frage, was die Betriebe zum Ausgangspunkt der Betrachtung (2007) kennzeichnet, die bis 2011 Öffnungsklauseln einführen, gehen wir in getrennten Schätzungen für entgelt- bzw. arbeitszeitrelevante Öffnungsklauseln nach analog zu unseren vorangegangenen Ausführungen. Für die Anwendung von Panelschätzverfahren ist die vorliegende Datenstruktur ungeeignet. Es gibt zwar mehrere Erhebungszeitpunkte mit der relevanten Information, die zeitlichen Abstände sind aber unregelmäßig und zu groß. Nichtsdestoweniger versuchen wir, die Panelstruktur des Datensatzes zumindest insoweit zu nutzen, als wir den Einstieg in die Nutzung von Öffnungsklauseln (zwischen 2007 und 2011) betrachten und die gewonnen Erkenntnisse in Bezug setzen zu interessierenden betrieblichen Charakteristika vor diesem Einstieg (also 2007). Indem die unabhängigen Variablen zeitlich vor der betrieblichen Implementierung der Öffnungsklauseln erfasst werden, stellen wir sicher, dass diese nicht selbst Resultat der Tarifunterschreitung sein können. Obwohl dieses Vorgehen eine Verbesserung gegenüber vorliegenden, auf Querschnittsdaten beruhenden Analysen darstellt, ist dadurch natürlich noch keinerlei Kausalitätsanspruch begründet. Die Interpretation der Ergebnisse fällt damit aber leichter.

Für die Analyse verwenden wir Probitschätzungen. Sie bieten sich an, wenn die abhängige Variable dichotome Ausprägungen (Implementierung von Öffnungsklauseln: ja/nein) aufweist. Die unabhängigen Variablen entstammen wie beschrieben der 2007er Welle des IAB-Betriebspanels. ${ }^{7}$

Als Kontrollgruppe (kein Einstieg in die Nutzung von Öffnungsklauseln) dienen uns diejenigen Betriebe, die weder 2007 noch 2011 Öffnungsklauseln nutzen. Aussteiger oder „Dauernutzer“ sowie Betriebe, denen das entsprechende Wis- sen fehlt, bleiben unberücksichtigt. Aufgrund der Überlegungen zur vermeintlichen Untererfassung der Existenz von Öffnungsklauseln (vgl. Abschnitt 3) rechnen wir zu unserer Kontrollgruppe auch solche Betriebe, die angeben, dass keine entsprechenden Regelungen in ihren Tarifverträgen existieren. Damit befinden sich in dieser Gruppe sicher auch Betriebe, die wegen der fehlenden rechtlichen Voraussetzungen gar keine Öffnungsklauseln anwenden können. Diese weite Definition der „Nicht-Einsteiger" bedeutet, dass unsere Kontrollgruppe u. U. „kontaminiert“ ist, insoweit sie Betriebe enthält, die - so für sie denn ein Tarifvertrag mit entsprechenden Regelungen gelten würde - vermutlich Öffnungsklauseln eingeführt hätten. Diese hinsichtlich ihrer Charakteristika im Grunde zu den „Einsteigern“ gehörenden Betriebe erschweren natürlich die klare Identifikation der Bedingungen, die vor der Implementierung von Öffnungsklauseln herrschen. Dies ist zwar unangenehm, stellt aber die erhaltenen Ergebnisse nicht infrage. Möglicherweise könnte sich aber bei einer besseren Abgrenzung der Kontrollgruppe ein klareres Bild ergeben.

Alternative Schätzungen mit einer entsprechend eingegrenzten Kontrollgruppe (Betriebe, die angeben, dass ihr Tarifvertrag Öffnungsklauseln beinhaltet, diese aber nicht genutzt werden) eignen sich unseres Erachtens allerdings nicht für einen Robustheitscheck. Mit Blick auf die vermutete starke Untererfassung der tarifgebundenen Betriebe mit Öffnungsklauseln ist davon auszugehen, dass es sich bei den Betrieben, die über solche Regelungen in ihrem Tarifvertrag berichten, um eine mehr oder weniger stark selektive Gruppe handelt. Betriebe, die sich bereits mit den Möglichkeiten auseinandergesetzt haben, die der Tarifvertrag in dieser Hinsicht bietet, befinden sich vermutlich viel eher in Umständen, die eine Nutzung dieser Möglichkeiten nahelegen. Die zu erwartenden Ergebnisse wären damit verzerrt. Gleichwohl versuchte Schätzungen zeichnen sich dann auch dadurch aus, dass rund zwei Drittel der Fälle nicht mehr berücksichtigt werden und sich so gut wie keine signifikanten Variablen zeigen. ${ }^{8}$

7 Dies geschieht mit einer Ausnahme. Die Information zur Wettbewerbssituation des Betriebs wurde im IAB-Betriebspanel 2007 nicht erhoben und wird deshalb aus der 2008er Welle verwendet. Wir halten dies für vertretbar, da wir davon ausgehen, dass sich die Konkurrenzsituation des Betriebs innerhalb eines Jahres nicht wesentlich verändern dürfte.

8 Abgesehen davon wurden Robustheitschecks in Form verschiedener Modellspezifikationen durchgeführt. Zum einen sind unterschiedliche Definitionen der unabhängigen Variablen geprüft worden, indem die parallele Nutzung der jeweils anderen Öffnungsklausel zugelassen bzw. ausgeschlossen wurde. Zum anderen sind Varianten gerechnet worden ohne die Exportvariable und den Anteil der Lohnkosten am Umsatz. Dadurch entfällt die Beschränkung auf die Umsatz-Betriebe, mit einem entsprechenden Zuwachs an Fällen. Bei allen Varianten ändern sich die Ergebnisse der (verbleibenden) Variablen nicht wesentlich. Die Ergebnisse dieser Schätzungen sind auf Nachfrage von den Autoren erhältlich. 


\subsection{Ergebnisse}

Inwieweit die gerade vorgestellten Variablen einen Einfluss auf die Wahrscheinlichkeit haben, dass Betriebe von Öffnungsklauseln tatsächlich Gebrauch machen, weist Tabelle 3 getrennt für entgelt- und arbeitszeitrelevante Regelungen aus. Die Tabelle bildet jeweils die marginalen Werte ab, also die Veränderung der Wahrscheinlichkeit, dass die abhängige Variable den Wert eins annimmt, bei einer Veränderung der unabhängigen Variable um eine Einheit (bei Dummyvariablen bei einem Wechsel von null auf eins).

Insgesamt zeigen sich in beiden Modellen nur wenige signifikante Variablen. Viele der diskutierten Determinanten haben offensichtlich keinen Einfluss auf die betriebliche Entscheidung, die tariflich vereinbarten Standards zu unterschreiten. Was sich aber herausstellt ist, dass es für beide Arten von Öffnungsklauseln durchaus unterschiedliche Faktoren sind, die das „Eintrittsgeschehen“ beeinflussen.

Für die betriebliche Umsetzung entgeltrelevanter Öffnungsklauseln ergibt sich folgendes Bild: Diese Betriebe klagen tatsächlich häufiger über eine schlechte Ertragslage. Entgegen unserer Erwartung, wird für die weitere Zukunft (Entwicklung der Beschäftigtenzahl in fünf Jahren) aber kein (deutlicher) Rückgang des Beschäftigtenstandes vermutet. Allerdings ist eine deutliche Unsicherheit erkennbar, überhaupt eine diesbezügliche Aussage treffen zu können. Die Lohnhöhe liegt hier zwar im Durchschnitt, der Lohnkostenanteil tendenziell sogar darunter, es werden aber für die kommenden Jahre Probleme mit zu hohen Lohnkosten erwartet. In dieses Bild passt auch, dass in diesen Betrieben signifikant seltener übertariflich bezahlt wird.

Bei den Nutzern von Lohnöffnungsklauseln handelt es sich eher um ältere Betriebe, die sich in vieler Hinsicht unauffällig verhalten. So bleiben sie mit ihren Flexibilitätsanstrengungen durchschnittlich. Gleiches gilt bei den Exportaktivitäten und der Investitions- und Innovationstätigkeit. Die Betriebsgröße hat keinen Einfluss, ebenso wenig die Branchenzugehörigkeit. Bemerkenswert ist, dass die Existenz eines Betriebsrats die betriebliche Umsetzung entgeltrelevanter Öffnungsklauseln deutlich wahrscheinlicher macht. Hier scheint die dem Betriebsrat in vielen Regelwerken zugewiesene Funktion bei der Konkretisierung der Tarifabweichungen durchzuschlagen. Darüber, ob diese Anforderung zulasten der Schutzfunktion des Betriebsrates gegenüber den Beschäftigten geht (Ellguth et al. 2012, S. 11), ließe sich an dieser Stelle nur spekulieren. Betriebe, die arbeitszeitrelevante Öffnungsklauseln nutzen, bewegen sich demgegenüber in einer etwas anderen Welt. Von wirtschaftlichen Schwierigkeiten ist hier nicht die Rede, ebenso wenig von schlechten Erwartungen oder Unsicherheiten hinsichtlich der längerfristigen Beschäftigungsentwicklung. Bei der Lohnhöhe zeigen sich keine Auffälligkeiten, es werden auch keine Probleme mit hohen Lohnkosten erwartet, und der Lohnkostenanteil ist tendenziell überdurchschnittlich. Es wird aber signifikant häufiger übertariflich bezahlt. Diese Betriebe stehen eher unter einem hohen Wettbewerbsdruck, was zu den verstärkten Exportaktivitäten passt.

Anders als zuvor sind hier überdurchschnittliche Flexibilitätsanstrengungen zu verzeichnen. Atypische Beschäftigungsformen sorgen für größere Flexibilitätspuffer. Darüber hinaus wird signifikant häufiger weitergebildet und so die interne Flexibilität erhöht. Hinzu kommen überdurchschnittliche Anstrengungen bei den Investitions- und Innovationsaktivitäten. Es passt ins Bild, dass es sich hier eher um größere (tendenziell jüngere) Betriebe handelt. Ebenso wie beim Einstieg in die Nutzung entgeltrelevanter Öffnungsklauseln spielt der Standort des Betriebs (in West- oder Ostdeutschland) keine Rolle.

Ein Ergebnis, das - vor allem mit Blick auf das Resultat bei den entgeltrelevanten Öffnungsklauseln - nicht so recht zu unseren Annahmen passt, ist der insignifikante Ko-

\section{TABELLE 3}

\section{Determinanten der Anwendung von Öffnungsklauseln zwischen 2007 und 2011'}

Probitschätzung (als Koeffizienten sind marginale Effekte ausgewiesen)

\begin{tabular}{|c|c|c|c|c|}
\hline \multirow{2}{*}{$\begin{array}{l}\text { Unabhängige Variablen } \\
\text { Ertragslage (sehr gut/gut =1) }\end{array}$} & \multicolumn{2}{|c|}{ Lohn-Öffnung } & \multicolumn{2}{|c|}{ AZ-Öffnung } \\
\hline & $-0,037^{*}$ & $(0,021)$ & $-0,003$ & $(0,022)$ \\
\hline $\begin{array}{l}\text { Beschäfigtenstand in } 5 \text { Jahren } \\
\text { ((deutlicher) Rückgang =1) }\end{array}$ & 0,004 & $(0,031)$ & 0,041 & $(0,036)$ \\
\hline $\begin{array}{l}\text { Beschäfigtenstand in } 5 \text { Jahren } \\
\text { (keine Aussage möglich }=1 \text { ) }\end{array}$ & $0,047^{*}$ & $(0,027)$ & 0,030 & $(0,028)$ \\
\hline Exportanteil & $-0,031$ & $(0,055)$ & $0,100 *$ & $(0,052)$ \\
\hline hoher Wettbewerbsdruck $(j a=1)(2008)$ & 0,012 & $(0,021)$ & $0,051^{*}$ & $(0,034)$ \\
\hline Investitionen ( $\mathrm{ja}=1)$ & 0,007 & $(0,028)$ & $0,077 * * *$ & $(0,024)$ \\
\hline Innovationen $(\mathrm{ja}=1)$ & 0,014 & $(0,024)$ & $0,046 *$ & $(0,024)$ \\
\hline Anteil befristet Beschäftigter & $-0,032$ & $(0,104)$ & 0,070 & $(0,100)$ \\
\hline Quote freier Mitarbeiter & $-0,082$ & $(0,580)$ & $1,040 * *$ & $(0,441)$ \\
\hline Quote Leiharbeiter & 0,193 & $(0,194)$ & $0,470 * * *$ & $(0,145)$ \\
\hline Weiterbildungsbetrieb $(j a=1)$ & $-0,007$ & $(0,027)$ & $0,067 * *$ & $(0,023)$ \\
\hline Lohn pro VZÄ (log) & 0,002 & $(0,028)$ & $-0,009$ & $(0,029)$ \\
\hline Anteil der Lohnkosten am Umsatz & $-0,346$ & $(0,571)$ & 0,383 & $(0,443)$ \\
\hline Übertarifliche Bezahlung ( $\mathrm{ja}=1$ ) & $-0,035^{*}$ & $(0,021)$ & $0,055^{* *}$ & $(0,022)$ \\
\hline Probleme mit Lohnkosten $(\mathrm{ja}=1)$ & $0,044 * *$ & $(0,022)$ & 0,012 & $(0,022)$ \\
\hline Betriebsrat $(j a=1)$ & $0,066 * *$ & $(0,030)$ & $-0,010$ & $(0,030)$ \\
\hline Beschäftigtenzahl (log) & 0,008 & $(0,009)$ & $0,030 * * *$ & $(0,010)$ \\
\hline Betriebsgründung vor $1990(\mathrm{ja}=1)$ & $0,048 *$ & $(0,025)$ & $-0,021$ & $(0,026)$ \\
\hline Managergeführt $(j a=1)$ & $-0,018$ & $(0,026)$ & $-0,038$ & $(0,025)$ \\
\hline Lage in Westdeutschland $(j a=1)$ & 0,009 & $(0,026)$ & 0,006 & $(0,027)$ \\
\hline Dummies für Wirtschaftszweige & \multicolumn{2}{|c|}{ nicht signifikant } & \multicolumn{2}{|c|}{ nicht signifikant } \\
\hline Wald Chi2 & \multicolumn{2}{|c|}{56,5} & \multicolumn{2}{|c|}{103,4} \\
\hline Pseudo R2 & \multicolumn{2}{|c|}{0,06} & \multicolumn{2}{|c|}{0,13} \\
\hline Fallzahl (Betriebe) & \multicolumn{2}{|c|}{1200} & \multicolumn{2}{|c|}{1025} \\
\hline
\end{tabular}

1 Basis: Privatwirtschaftliche Betriebe ab 5 Beschäftigte mit Tarifbindung, ohne Landwirtschaft und Organisationen ohne Erwerbszweck.

Anmerkung: $* * * * * * *$ signalisieren einen signifikant positiven bzw. negativen Zusammenhang auf dem 1\%-/5\%-/10\%-Niveau. Standardfehler in Klammern.

Quelle: IAB-Betriebspanel (2007; 2011). 
effizient der Betriebsratsvariablen. Würde sich tatsächlich die beschriebene Funktionszuweisung im Zuge der Umsetzung von Öffnungsklauseln bemerkbar machen, sollte sich das bei arbeitszeitrelevanten Regelungen eigentlich stärker auswirken, da hier der gegenläufige Einfluss einer eventuellen Schutzfunktion geringer sein sollte. Hier bieten sich zwei Erklärungen für das unerwartete Ergebnis an. Zum einen wäre denkbar, dass in Lohnöffnungsklauseln häufiger der Betriebsrat als Instanz zur Aushandlung entsprechender Betriebsvereinbarungen adressiert wäre als in Arbeitszeitöffnungsklauseln. Dies ließe sich aber nur mit einer entsprechenden - extrem aufwendigen - Analyse aller relevanten Tarifvertragstexte verifizieren. Zum anderen ließe sich argumentieren, dass die Existenz eines Betriebsrats auch eine genuine Push-Funktion bei der Nutzung entgeltrelevanter Öffnungsklauseln ausüben kann, indem er die Funktion erfüllt, die Beschäftigten von der Notwendigkeit drastischer, d.h. die Entlohnung betreffender Maßnahmen zu überzeugen. In Betrieben ohne Betriebsrat würden die Geschäftsleitungen danach eher vor solchen Einschnitten zurückschrecken, da es an deren glaubhafter Vermittlung durch die Arbeitnehmervertretungen fehlt.

\section{Fazit}

In den meisten Branchentarifverträgen finden sich heute Flexibilisierungs- und Differenzierungsmöglichkeiten, die es den Betrieben erlauben, unter bestimmten Bedingungen von tariflichen Standards nach unten abzuweichen. Ziel der vorliegenden Analyse war, Determinanten der Implementierung solcher Öffnungsklauseln auszuleuchten. Um den Einstieg in die Nutzung analysieren zu können, wurden Betriebe des IABBetriebspanels betrachtet, die sowohl 2007 als auch 2011 existierten. Etwa 10 \% dieser Betriebe führten bis 2011 Entgeltöffnungsklauseln und rund $13 \%$ solche zu Arbeitszeiten ein.

Für beide Formen von Öffnungsklauseln haben wir, geleitet durch theoretische Überlegungen, getrennte Schätzungen durchgeführt. Die Schätzergebnisse bestätigen, dass diese Vorgehensweise angemessen war - selbst wenn sich in beiden Modellen nur relativ wenige signifikante Einflussfaktoren zeigen.

(1) Insgesamt wird deutlich, dass Lohnöffnungsklauseln eher in älteren Betrieben mit unsicherer Zukunftserwartung und schlechter Ertragslage eingesetzt werden, während arbeitszeitrelevante Regelungen offensichtlich eher den Rahmen erweitern für Flexibilisierungsanstrengungen in Betrieben, die sich in einem hochkompetitiven Umfeld bewegen.

(2) Ein unklares Bild liefern die Ergebnisse zur Rolle des Betriebsrats. Hier wirken vermutlich verschiedene Faktoren in entgegengesetzte Richtung. Der Umstand, dass in vielen tariflichen Öffnungsklauseln die Existenz eines Betriebsrates als zwingende Voraussetzung für die betriebliche Nut- zung von Öffnungsklauseln gefordert wird, dürfte, so unsere Vorannahme, ihre Anwendung in Betriebsratsbetrieben wahrscheinlicher machen. Die Schutzfunktion des Betriebsrats sollte hingegen einen dämpfenden Einfluss ausüben. Vor diesem Hintergrund überrascht das Ergebnis, dass die Existenz eines Betriebsrats gerade die Nutzung von Entgeltöffnungsklauseln wahrscheinlicher macht, während sich für die Implementierung von vom Tarifvertrag abweichenden Arbeitszeitregelungen kein Zusammenhang zeigt. Hier gibt es offensichtlich weiteren Forschungsbedarf.

(3) Die Ergebnisse unserer Schätzungen bestätigen frühere empirische Studien nur teilweise. Auch Kohaut/Schnabel (2007, S.38) finden einen negativ signifikanten Zusammenhang zwischen einer schlechten wirtschaftlichen Situation und entsprechenden Erwartungen beim Einsatz von Entgeltöffnungsklauseln. Die in dieser Studie gleichfalls festgestellten Unterschiede zwischen West- und Ostdeutschland sind in unseren Auswertungen allerdings nicht (mehr) nachzuweisen. Es liegt nahe, diese Diskrepanz als Zeichen einer weitgehenden Angleichung in der Nutzung von Öffnungsklauseln in beiden Landesteilen zu verstehen, bei fortdauernden Unterschieden in der Verbreitung von Branchentarifen.

(4) Das Segment der tarifgebundenen Betriebe lässt sich nach unseren Ergebnissen in vier Gruppen unterteilen:

Erstens gibt es diejenigen Betriebe, für die tarifliche Öffnungsklauseln kein Thema sind. Dazu gehören die, die nicht wissen, ob es entsprechende Regelungen in dem für sie geltenden Tarifvertrag gibt (und das auch in der Befragung angeben). Hierzu zählt aber auch die nicht unerhebliche Gruppe von Betrieben, die in der Erhebung antworten, dass keine Öffnungsklauseln für ihren Betrieb existieren, obwohl es nach den Ergebnissen anderer Datenquellen diese in den meisten Tarifvertragswerken geben sollte. Inwieweit sich aus Unkenntnis der einschlägigen Regelungen auf die Abwesenheit von Problemen mit den Standards des jeweiligen Branchentarifs schließen lässt, bleibt letztlich Spekulation; auch wenn zu vermuten ist, dass sich die betrieblichen Entscheidungsträger im gegenteiligen Fall über die Flexibilitätsspielräume, die Öffnungsklauseln bieten, informieren würden.

Zur zweiten Gruppe zählen Betriebe, die mit den tariflichen Regelungen vertraut sind, über die Existenz entsprechender Öffnungsklauseln Auskunft geben können, aber keine Notwendigkeit sehen, diese in der betrieblichen Praxis umzusetzen.

Die tatsächlichen Nutzer unterteilen sich wiederum in die dritte Gruppe der im harten (internationalen) Wettbewerb agierenden Unternehmen, die alle zur Verfügung stehenden Instrumente zur Steigerung ihrer Anpassungsfähigkeit einsetzen, wozu auch die Anwendung von Arbeitszeitöffnungsklauseln gehört.

Schließlich gibt es als vierte Gruppe diejenigen Betriebe, die Öffnungsklauseln als Mittel zur Abwendung einer betrieblichen Notlage nutzen und damit ihre Lohnkosten reduzieren. Was ihre Relevanz betrifft, so sehen sich immerhin 
in dem vierjährigen Betrachtungszeitraum $10 \%$ der tarifgebundenen Betriebe (Privatwirtschaft ab fünf Beschäftigte) genötigt, in die Nutzung von Entgeltöffnungsklauseln einzusteigen. Inwieweit diese Betriebe sich ohne die Möglichkeit der Tarifabweichung tatsächlich in ihrer Existenz bedroht sehen oder ihr Heil in einem Austritt aus dem Arbeitgeberverband suchen würden, muss offen bleiben. Zumindest was die letzte Frage angeht, gibt es in der vorliegenden Literatur keine eindeutigen Hinweise auf eine solche Strategie (vgl. Ellguth/Kohaut 2008).

(5) Was die Faktoren angeht, die zur Implementierung von Öffnungsklauseln führen, bleiben unsere Ergebnisse insgesamt eher unbefriedigend, obwohl wir Längsschnittdaten, mit denen eine klare zeitliche Abfolge modelliert werden kann, verwenden. Neben den Problemen, die sich aus der schwierigen Definition der Kontrollgruppe ergeben, könnte dies daran liegen, dass Entscheidungen zur Nutzung von Öffnungsklauseln im Betrieb möglicherweise entlang anderer Kriterien getroffen werden, als sie eine Betriebsbefragung erfassen kann. Allerdings spricht auch einiges dafür, dass viele der Betriebe eher unaufgeregt mit dem Thema umgehen und - soweit sie entsprechende Kenntnis haben - einfach den erweiterten Regelungsrahmen, den Öffnungsklauseln bieten, ausschöpfen.

\section{LITERATUR}

Bellmann, L./Gerlach, K./Meyer, W. (2008): Company-level pacts for employment, in: Journal of Economics and Statistics $228(5+6)$, S. 533-553 Bellmann, L./Gerner, H.-D. (2012a): Company-level pacts for employment in the global crisis 2008/2009. First evidence from representative German establishment-level panel data, in:The International Journal of Human Resource Management 23 (16), S. 3375-3396

Bellmann, L./Gerner, H.-D. (2012b): Further training and company-level pacts for employment in Germany, in: Jahrbücher für Nationalökonomie und Statistik 232 (2), S. 98-115

Bernard, A./Wagner, J. (1997): Export and success in German manufacturing in: Weltwirtschaftliches Archiv 133 (1), S. 134-157

Berthold, N./Brischke, M./Stettes, O. (2003): Betriebliche Bündnisse für Arbeit - Gratwanderung zwischen Tarifbruch und Tariftreue, Wirtschaftswissenschaftliche Beiträge des Lehrstuhls für Volkswirtschaftslehre, Wirtschaftsordnung und Sozialpolitik: Universität Würzburg, Working Paper (64)

Bispinck, R. (2004): Kontrollierte Dezentralisierung derTarifpolitik - Eine schwierige Balance, in: WSI-Mitteilungen 57 (5), S. 237-245,

http://www.boeckler.de/wsi_24421_24430.htm

Bispinck, R./WSI-Tarifarchiv (2004): Kontrollierte Dezentralisierung. Eine Analyse der tariflichen Öffnungsklauseln in 80 Tarifbereichen, Elemente qualitativerTarifpolitik (55), Düsseldorf

Bispinck, R./Schulten, Th. (2011): Sector-level bargaining and possibilities for deviations at company level: Germany. European Foundation for the Improvement of Living and Working Conditions, http://www.eurofound.europa.eu/ publications/htmlfiles/ef10874.htm (letzter Zugriff: 16.04.2013)

Bosch, A./Ellguth, P./Schmidt, R./Trinczek, R. (1999): Betriebliches Interessenhandeln: Zur politischen Kultur der Austauschbeziehungen zwischen Management und Betriebsrat in der westdeutschen Industrie, Opladen

Brändle, T./Heinbach, W. D. (2013): Opening clauses in collective bargaining agreements: More flexibility to save jobs?, in: Review of Economics 64 (2), S. 159-192 Brändle, T./Heinbach W. D./Mauer, M. (2011): Tarifliche Öffnung in Deutschland: Ausmaß, Determinanten, Auswirkungen, in: Zeitschrift für Arbeitsmarktforschung $44(1+2)$, S. $163-172$

Dütsch, M./Struck, O. (2007): Interne und externe Flexibilität. Eine Analyse von Personalanpassungsformen anhand des IAB-Betriebspanels 2007: Universität Bamberg, Working Paper (2)

Ellguth, P./Kohaut, S. (2008): Ein Bund fürs Überleben? Betriebliche Vereinbarungen zur Beschäftigungs- und Standortsicherung, in: Industrielle Beziehungen 15 (3), S. 209-232
Ellguth, P./Gerner, H.-D./Stegmeier, J. (2012): Wage effects of works councils and opening clauses: The German case, in: Economic and Industrial Democracy, Online First

Ellguth, P./Kohaut, S./Möller, I. (2013): The IAB Establishment Panel - methodological essentials and data quality, in: Journal of Labour Market Research, Online First 10.1007/s12651-013-0151-0

Fischer, G./Janik, F./Müller, D./Schmucker, A. (2008): Das IAB-Betriebspanel von der Stichprobe über die Erhebung bis zur Hochrechnung, FDZ-Methodenreport 01/2008, Nürnberg

Franz, W./Pfeifer F. (2003): Zur ökonomischen Rationalität von Lohnrigiditäten aus der Sicht von Unternehmen, in: Jahrbücher für Nationalökonomie und Statistik 223 (1), S. 23-57

Garloff, A./Gürtzgen, N. (2012): Collective wage contracts, opt-out clauses and firm wage-differentials: Evidence from linked employer employee data, in: Industrial Relations 51 (3), S. $731-748$

Heinbach, W. D. (2005): Ausmaß und Grad der tarifvertraglichen Öffnung, IAW Report 2005 (2), S. 51-70

Heinbach, W. D./Schröpfer, S. (2008): What a difference trade makes - export activity and the flexibility of collective bargaining agreements, in: Zeitschrift für Arbeitsmarktforschung 41 (2+3), S. 287-303

Hohendanner, Ch./Bellmann, L. (2006): Interne und externe Flexibilität, in: WSIMitteilungen 59 (5), S. 241-246, http://www.boeckler.de/wsi_24735_24745.htm Kohaut, S./Schnabel, C. (2007): Tarifliche Öffnungsklauseln - Verbreitung, Inanspruchnahme und Bedeutung, in: Sozialer Fortschritt 56 (2), S. 33-40 Massa-Wirth, H. (2007): Zugeständnisse für Arbeitsplätze? Konzessionäre Beschäftigungsvereinbarungen im Vergleich Deutschland - USA, Berlin Schnabel, C. (2003): Tarifpolitik unter Reformdruck: Bertelsmann Stiftung, Gütersloh

Spindler, M. (2007): Determinanten der Einführung von tariflichen Öffnungsklauseln - Eine empirische Analyse mit Hilfe von Panel-Logit und Panel-ProbitModellen, Diplomarbeit, Fakultät Wirtschafts- und Sozialwissenschaften, Universität Hohenheim

Wagner, J. (2007): Exports and productivity in Germany, in: Applied Economics Quarterly 53 (4), S. 353-373

Statistisches Bundesamt (2012): https://www.destatis.de/DE/ZahlenFakten/ GesamtwirtschaftUmwelt/VerdiensteArbeitskosten/Tarifverdienste/TDB/TDB/ TarifdatenbankIF.html (letzter Zugriff 16.04.2013)

\section{AUTOREN}

PETER ELLGUTH, ist wissenschaftlicher Mitarbeiter im Institut für Arbeitsmarktund Berufsforschung (IAB) der Bundesagentur für Arbeit im Forschungsbereich "Betriebe und Beschäftigung". Arbeitsschwerpunkte: Arbeitsbeziehungen und betriebliche Arbeitszeitpolitik.

peter.ellguth@iab.de

SUSANNE KOHAUT, Dr., ist wissenschaftliche Mitarbeiterin im IAB im Forschungsbereich „Betriebe und Beschäftigung”. Arbeitsschwerpunkte: Arbeitsbeziehungen und Betriebsgründungen.

susanne.kohaut@iab.de 